\title{
Mass Spectra of Di-baryonic Systems in Charm sector
}

\section{P. Rathaud, Zalak Shah ${ }^{* \dagger}$ and Ajay Kumar Rai}

Sardar Vallabhbhai National Institute of Technology-Surat, Gujarat-395007, India.

E-mail: dharmeshphy@gmail.com

In the potential model frame work, we study the S-wave molecular like structure of the Hexaquark systems which consist baryon-baryon or baryon-antibaryon as a constituents. The mass spectra of the $\Sigma_{c}-\Sigma_{c}$ and $\Sigma_{c}-\overline{\Sigma_{c}}$ are predicted. By taking the deuteron as an approximation for our model calculation, the S-wave mass spectra of the molecular Hexaquark system are extracted. The One Boson Exchange Potential plus screen Yukawa-like potential is being used for the inter baryonbaryon(antibaryon) interaction. The calculated mass spectra with possible quantum numbers are presented here.

XVII International Conference on Hadron Spectroscopy and Structure - Hadron2017

25-29 September, 2017

University of Salamanca, Salamanca, Spain

\footnotetext{
* Speaker.

${ }^{\dagger}$ Poster presenter.
} 


\section{Introduction}

The underlying theory of Quantum Chromodynamics (QCD) is being disputed from a long time by the absence of multi-quark states, hence search of these predicted exotic states is an effortful theoretical and experimental task in the field of fundamental physics. Specially, the hadronic molecules which is the subject of this work is also an interesting issue in the field of strong interaction, with regards to its large theoretical possibilities, lack of experimental observation as well as the difficulties with their identification. The another issue with hadronic molecules is their interaction potential which is largely unknown yet and we still do not know that how these loosely bound hadrons are interacting. In the non-strange sector, the deuteron is the only known experimentally candidate as a hadronic molecule. Apart from deuteron, we are still searching other strong hadronic molecular candidate. There are large numbers of the hadronic molecules have been predicted in the various theoretical approaches in which H-dibaryon (in strange sector) is the most promising candidate with deep binding [1-11]. In this work, we have presented mass spectra of the possible $\Sigma_{c}-\Sigma_{c}$ and $\Sigma_{c}-\overline{\Sigma_{c}}$ as di-hadron molecule. We have approximated the interaction potential as a s-wave One Boson Exchange plus screen Yukawa like potential. We proposed that the two hadron in the loosely bound hadronic molecule is experienced the dipole like interaction. Here, we present the only few results while the detail study and results are preserved for subsequent publication.

\section{Theoretical Framework}

To extract the S-wave ground state energy of the di-hadronic system, we employ the variational scheme to determine the expectation value of the Hamiltonian, using hydrogen like trial wave function. The Hamiltonian of the system read [5, 8-11]

$$
H=\sqrt{P^{2}+m_{h 1}^{2}}+\sqrt{P^{2}+m_{h 2}^{2}}+V_{h h}
$$

Here, $m_{h 1}$ and $m_{h 2}$ are masses of the constituent hadron while $\mathrm{P}$ is relative momentum. $V_{h h}$ is the interaction potential. To incorporate the relativistic effect due to the light quarks, we have expanded the kinetic energy term of the Hamiltonian up to $\mathscr{O}\left(P^{6}\right)$. The binomial expansion of the kinetic energy term is given as

$$
K . E .=\frac{P^{2}}{2}\left(\frac{1}{m_{h 1}}+\frac{1}{m_{h 2}}\right)-\frac{P^{4}}{8}\left(\frac{1}{m_{h 1}^{3}}+\frac{1}{m_{h 2}^{3}}\right)+\frac{P^{6}}{16}\left(\frac{1}{m_{h 1}^{5}}+\frac{1}{m_{h 2}^{5}}\right)+\mathscr{O}\left(P^{8}\right)
$$

The interaction potential could expressed as

$$
V_{h h}=V_{O B E}+V_{Y}
$$

where, $V_{O B E}$ is the One Boson Exchange Potential [7]

$$
V_{O B E}=V_{p s}+V_{s}+V_{v}
$$

the individual s-wave one meson exchange interaction potential expressed as [7]

$$
V_{p s}=\frac{1}{12}\left[\frac{g_{\pi q q}^{2}}{4 \pi}\left(\frac{m_{\pi}}{m}\right)^{2} \frac{e^{-m_{\pi} r_{12}}}{r_{12}}\left(\tau_{1} \cdot \tau_{2}\right)+\frac{g_{\eta q q}^{2}}{4 \pi}\left(\frac{m_{\eta}}{m}\right)^{2} \frac{e^{-m_{\eta} r_{12}}}{r_{12}}\right]\left(\sigma_{1} \cdot \sigma_{2}\right)
$$




$$
\begin{gathered}
V_{s}=-\frac{g_{\sigma q q}^{2}}{4 \pi} m_{\sigma}\left[1-\frac{1}{4}\left(\frac{m_{\sigma}}{m}\right)^{2}\right] \frac{e^{-m_{\sigma} r_{12}}}{m_{\sigma} r_{12}}+\frac{g_{\delta q q}^{2}}{4 \pi} m_{\delta}\left[1-\frac{1}{4}\left(\frac{m_{\delta}}{m}\right)^{2}\right] \frac{e^{-m_{\delta} r_{12}}}{m_{\delta} r_{12}}\left(\tau_{1} \cdot \tau_{2}\right) \\
V_{v}=\frac{g_{\omega q q}^{2}}{4 \pi}\left(\frac{e^{-m_{\omega} r_{12}}}{r_{12}}\right)+\frac{1}{6} \frac{g_{\rho q q}^{2}}{4 \pi} \frac{1}{m^{2}}\left(\tau_{1} \cdot \tau_{2}\right)\left(\sigma_{1} \cdot \sigma_{2}\right)\left(\frac{e^{-m_{\rho} r_{12}}}{r_{12}}\right)
\end{gathered}
$$

and the additional screen Yukawa-like potential introduced as

$$
V_{Y}=-\frac{\alpha_{s}}{r_{12}} e^{\frac{-c^{2} r_{12}^{2}}{2}}
$$

In the variational scheme, we have used the Hydrogenic trial wave function, namely

$$
R_{n l}\left(r_{12}\right)=\left(\frac{\mu^{3}(n-l-1) !}{2 n(n+l)^{3} !}\right)^{\frac{1}{2}}\left(\mu r_{12}\right)^{l} e^{\frac{-\mu r_{12}}{2}} L_{n-l-1}^{2 l+1}\left(\mu r_{12}\right)
$$

where $L_{n-l-1}^{2 l+1}\left(\mu r_{12}\right)$ is the Laguerre polynomial and $\mu$ is the variational parameter.

$$
H \psi=E \psi \quad \text { and } \quad\langle K . E .\rangle=\frac{1}{2}\left\langle\frac{r_{12} d V\left(r_{12}\right)}{d r_{12}}\right\rangle
$$

The ground state energy of the low-lying system are calculated by obtaining the expectation value of the Hamiltonian. The variational parameter $(\mu)$ is determined for each state by using the Virial theorem.

\section{Result, Discussion and Summery}

We have fitted the parameters of the potential to get binding energy of the deuteron and than

\begin{tabular}{|c|c|c|c|c|c|c|c|c|c|}
\hline $\begin{array}{c}\text { System } \\
{\left[I\left(J^{P}\right)\right]^{Q}\left[I\left(J^{P}\right)\right]^{Q}}\end{array}$ & $I\left(J^{P}\right)$ & $\begin{array}{c}\mu \\
\mathrm{GeV}\end{array}$ & $\begin{array}{c}\mu \\
\mathrm{GeV}\end{array}$ & $\begin{array}{l}\text { B.E. } \\
\mathrm{MeV}\end{array}$ & $\begin{array}{l}\text { B.E. } \\
\text { MeV }\end{array}$ & $\begin{array}{l}\text { Mass } \\
\mathrm{GeV}\end{array}$ & $\begin{array}{l}\text { Mass } \\
\mathrm{GeV}\end{array}$ & $\begin{array}{l}\sqrt{r^{2}} \\
\mathrm{fm}\end{array}$ & $\begin{array}{l}\sqrt{r^{2}} \\
\mathrm{fm}\end{array}$ \\
\hline \multirow{5}{*}[1(\frac{1}{2}^{+})]{$^{0}-\left[1\left(\frac{1}{2}^{+}\right)\right]^{0}$} & & 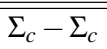 & $\overline{\overline{\Sigma_{c}}-\overline{\Sigma_{c}}}$ & 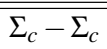 & $\overline{\overline{\Sigma_{c}-\overline{\Sigma_{c}}}}$ & 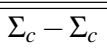 & $\overline{\overline{\Sigma_{c}-\overline{\Sigma_{c}}}}$ & $\overline{\overline{\Sigma_{c}-\Sigma_{c}}}$ & $\overline{\overline{\Sigma_{c}-\overline{\Sigma_{c}}}}$ \\
\hline & $0\left(0^{+}\right)$ & 0.3271 & 0.3271 & -12.86 & -12.86 & 4.894 & 4.894 & 1.04 & 1.04 \\
\hline & $1\left(0^{+}\right)$ & 0.3806 & 0.3606 & -02.14 & -01.06 & 4.905 & 4.906 & 0.89 & 0.94 \\
\hline & $0\left(1^{+}\right)$ & 0.3593 & 0.3424 & -14.66 & -13.73 & 4.892 & 4.893 & 0.95 & 0.99 \\
\hline & $1\left(1^{+}\right)$ & 0.3686 & 0.3686 & -01.49 & -01.49 & 4.905 & 4.905 & 0.92 & 0.92 \\
\hline \multirow{4}{*}[1(\frac{3}{2}^{+})]{$^{0}-\left[1\left(\frac{3}{2}^{+}\right)\right]^{0}$} & $0\left(0^{+}\right)$ & 0.3288 & 0.3288 & -12.85 & -12.85 & 5.024 & 5.024 & 1.03 & 1.03 \\
\hline & $1\left(0^{+}\right)$ & 0.3816 & 0.3622 & -02.06 & -01.03 & 5.035 & 5.036 & 0.89 & 0.94 \\
\hline & $0\left(3^{+}\right)$ & 0.0008 & 0.2593 & +05.50 & -08.43 & 5.043 & 5.029 & 380 & 1.31 \\
\hline & $1\left(3^{+}\right)$ & 0.3263 & 0.3263 & +01.06 & +01.06 & 5.038 & 5.038 & 1.04 & 1.04 \\
\hline \multirow{4}{*}[1(\frac{1}{2}^{+})]{$^{0}-\left[1\left(^{3}{ }^{+}\right)\right]^{0}$} & $0\left(1^{+}\right)$ & 0.3596 & 0.3431 & -14.61 & -13.71 & 4.957 & 4.958 & 0.95 & 0.99 \\
\hline & $1\left(1^{+}\right)$ & 0.3822 & 0.3822 & -02.48 & -02.48 & 4.970 & 4.970 & 0.89 & 0.89 \\
\hline & $0\left(2^{+}\right)$ & 0.0009 & 0.0009 & +05.50 & +05.50 & 4.978 & 4.978 & 371 & 371 \\
\hline & $1\left(2^{+}\right)$ & 0.3603 & 0.4284 & -01.24 & -04.94 & 4.971 & 4.967 & 0.94 & 0.79 \\
\hline
\end{tabular}
fixed them for further calculation. We have taken $\Sigma_{c}\left(\frac{1}{2}\right)^{0}$ and $\Sigma_{c}\left(\frac{3}{2}\right)^{0}$ as a constituent, where the

Table 1: Mass Spectra, root mean square radius of $\Sigma_{c}-\Sigma_{c}$ and $\Sigma_{c}-\overline{\Sigma_{c}}$. Mass of the baryon is taken from PDG [12]. Here, I, Q, J, P are isospin, charge, total angular momentum and parity quantum numbers, respectively. 
masses and quantum numbers are taken from PDG [12]. We have found the bound state (almost equal in both case of $\left.\Sigma_{c}-\Sigma_{c} \& \Sigma_{c}-\overline{\Sigma_{c}}\right)$ for $(\mathrm{I}, \mathrm{S})=(0,1),(0,0)$ and $(1,1)$, in which channel $(0,1)$ is being the strongest bound state. While the channel $(\mathrm{I}, \mathrm{S})=(1,1)$ is found to be a very shallow bound state, very near to threshold. Whereas, the channel $(\mathrm{I}, \mathrm{S})=(0,3)$ is found bound for $\Sigma_{c}-\overline{\Sigma_{c}}$ while it is unbound in the case of $\Sigma_{c}-\Sigma_{c}$. The channel $(\mathrm{I}, \mathrm{S})=(1,3)$ is found unbound but it is just above the threshold. Moreover, for the channel $(I, S)=(2,0),(2,1)$ and $(2,3)$ we have not found any acceptable solution. We have found the solution such that we get extremely over bounded states which seems unphysical. Thus we have considered these solution as forbidden cases and omitted from Table. In Ref. [3], N. Lee et.al., has studied the possible molecular states composed of heavy baryons in the one boson exchange scheme. In summery, we have calculated the mass spectra of the $\Sigma_{c}-\Sigma_{c}$ $\& \Sigma_{c}-\overline{\Sigma_{c}}$ bound states which seems to be deuteron like structure. In the present formalism, it has been interesting to search other possible di-baryonic bound states. We will present the detail study of the di-baryonic and meson-baryon bound states with the approximated interaction in the subsequent future publication.

\section{References}

[1] Takashi Inoue et. al. (HALQCD Collaboration), Bound H Dibaryon in Flavor SU(3) Limit of Lattice QCD, Phys. Rev. Lett. 106, 162002 (2011).

[2] S. R. Beane et. al. (NPLQCD Collaboration), Evidence for a Bound H Dibaryon from Lattice QCD, Phys. Rev. Lett. 106, 162001 (2011).

[3] N. Lee, Zhi-Gang Luo, Xiao-Lin Chen, and Shi-Lin Zhu, Possible deuteronlike molecular states composed of heavy baryons, Phys. Rev. D 84, 014031 (2011).

[4] S. Patel and P. C. Vinodkumar, Tetraquark states in the bottom sector and the status of the $Y_{b}(10890)$ state, Eur. Phys. J C 76, 356 (2016).

[5] A. K. Rai, J. N. Pandya and P. C. Vinodkumar, Low-lying di-hadronic states in relativistic harmonic model, Ind. J Phys. 80, 387 (2006).

[6] A. K. Rai et. al., Multiquark states as di-hadronic molecules, Nucl. Phys. A 782, 406 (2007).

[7] R. Machleidt, High-precision, charge-dependent Bonn nucleon-nucleon potential, Phys. Rev. C 63, 024001 (2001).

[8] D. P. Rathaud and A. K. Rai, Dimesonic states with the heavy-light flavour mesons, Eur. Phys. J Plus 132, 370 (2017).

[9] D. P. Rathaud and A. K. Rai, Mass spectra of dimesonic states in light flavour sector, Indian J. Phys. 90, 1299 (2016).

[10] A. K. Rai and D. P. Rathaud, The mass spectra and decay properties of dimesonic states, using the Hellmann potential, Eur. Phys. J C 75, 462 (2015).

[11] D. P. Rathaud and A. K. Rai, Dimesonic states in the heavy-light meson sector, AIP Conf. Proc. 1728, 020115 (2015); Masses of di-mesonic molecular states, EPJ Web Conf. 9505013 (2015).

[12] C. Patrignani et al. (Particle Data Group), The Review of Particle Physics (2017), Chin. Phys. C, 40, 100001 (2016). 